Marla Weihs ${ }^{1}$

Frédéric Mertens ${ }^{2}$
${ }^{1}$ Departamento de Ciências Biológicas, Campus de Alta Floresta, Universidade do Estado de Mato Grosso. Rodovia MT 208 Km 147, Jardim Tropical. 78580-000 Alta Floresta MT.

marlaweihs@gmail.com

${ }^{2}$ Centro de

Desenvolvimento Sustentável, Universidade de Brasília.

\section{Os desafios da geração do conhecimento em saúde ambiental: uma perspectiva ecossistêmica}

\author{
Challenges for knowledge generation in environmental health: \\ an ecosystemic approach
}

\begin{abstract}
This article examines opportunities and limitations regarding knowledge generation in the field of environmental health. The contention is that understanding the complexity of factors that determine the health of humans and ecosystems requires a redefinition of the traditional distribution of roles and responsibilities in scientific research. These research practices involve inter and transdisciplinary approaches and the application of an ecosystemic approach (ecohealth). Challenges and opportunities associated to the application of inter and transdisciplinarity in environmental health problems are discussed and illustrated by two case studies that use an ecohealth approach: a project on the contamination and exposure to mercury in the Brazilian Amazon, and another on the urban transmission of echinococcosis in Nepal. In the conclusion, the potential benefits of using an ecohealth approach in overcoming the limitations of unidisciplinary practices and in taking advantage of local knowledge and participation is stressed.
\end{abstract}

Key words Interdisciplinarity, Transdisciplinarity, Ecosystemic approach, Environmental health, CARUSO project, Kathmandu
Resumo $O$ artigo explora as oportunidades e as limitações da geração de conhecimento no campo da saúde ambiental. Argumenta que a compreensão da complexidade dos fatores que condicionam a saúde humana e dos ecossistemas demanda redefinições na tradicional distribuição de papéis e responsabilidades na pesquisa científica. Estas práticas de pesquisa encerram enfoques inter $e$ transdisciplinares e a aplicação de uma abordagem ecossistêmica (ecosaúde). Desafios e oportunidades da aplicação da inter e transdisciplinaridade a problemáticas de saúde ambiental são discutidos e ilustrados por meio de dois estudos de caso que utilizam uma abordagem ecosaúde: uma experiência brasileira que trata da contaminação e exposição ao mercúrio na Amazônia, e outra nepalense, sobre a transmissão urbana de equinococose. Concluimos apresentando o potencial de uma abordagem ecosaúde na superação dos limites das práticas unidisciplinares e na valorização dos saberes e da participação local.

Palavras-chave Interdisciplinaridade, Transdisciplinaridade, Abordagem ecossistêmica, Saúde ambiental, Projeto Caruso, Katmandu 


\section{Introdução}

Os fatores ambientais afetam a saúde humana. Este é um consenso amplamente difundido, mesmo que pouco compreendido. Os desafios de sua compreensão situam-se na complexa matriz de elementos que determinam e integram a saúde. São fatores como geografia, clima, trabalho, alimentação, educação, habitação, cultura e valores éticos. Há ainda as capacidades e potencialidades de cada indivíduo, em seus aspectos físicos, fisiológicos, psicológicos, sociais, entre outros. Esses elementos, em conjunto, dão a condição do que podemos chamar de bem-estar, felicidade ou, traduzidos numa concepção mais conhecida, um completo estado de saúde ${ }^{1,2}$.

Se a compreensão da complexidade dessas interações demanda, por um lado, a integração de disciplinas das ciências naturais e humanidades, por outro requer a participação da sociedade na formulação e implementação de estratégias de gestão dos problemas. Neste contexto, dois desafios se tornam primordiais à geração de conhecimento no campo da saúde ambiental. O primeiro implica o emprego de uma abordagem interdisciplinar, por meio do diálogo entre disciplinas específicas da ciência ${ }^{3,4}$. O segundo concerne à transdisciplinaridade, enfoque que visa integrar os saberes de atores locais na pesquisa e na gestão da saúde humana e do ecossistema ${ }^{5,6}$.

Em ambos os casos, a formação de equipes e a rotina de trabalho requerem um pesquisador que esteja em constante reflexão autocrítica. Sobretudo, demandam um método que atenda as especificidades da pesquisa e da gestão dos problemas. Neste contexto, têm sido empregados os enfoques inter a transdisciplinares de pesquisa, no âmbito de uma abordagem ecossistêmica para a saúde humana (ecosaúde). Trata-se de um método de investigação que pretende, por um lado, articular diferentes campos teóricos para entender as conexões históricas entre a natureza, a sociedade e a saúde ${ }^{3,7}$. Por outro, se concretiza num movimento que vincula o conhecimento do problema e a sua resolução, em cooperação com as populações envolvidas ${ }^{8}$.

Os desafios desta prática são a questão central deste texto, que busca sistematizar categorias-chaves e dar atenção a oportunidades e limitações da geração de conhecimento no campo da saúde ambiental. O propósito não é abordar a temática pela simples crítica, mas destacar como a área vem sendo construída e para onde apontam os resultados das experiências. Neste sentido, se buscou refletir e apresentar uma nova pers- pectiva sobre a questão da geração de conhecimento no campo da saúde ambiental. Os desafios da inter e transdisciplinaridade e da ecosaúde são os principais aspectos abordados.

O texto está dividido em quatro seções. A primeira traça o caminho de construção dos conceitos no campo da saúde ambiental. Na seguinte, é feita a distinção entre a inter e transdisciplinaridade na geração do conhecimento neste campo. Os principais desafios enfrentados pelo pesquisador individual e pelas equipes de pesquisa são abordados na terceira seção. Na quarta, são examinados os avanços científicos e sociais no âmbito de duas experiências de pesquisa inter e transdisciplinares desenvolvidas no escopo da ecosaúde. O potencial deste enfoque, na superação dos limites das abordagens unidisciplinares e na valorização dos saberes e da participação local, numa perspectiva de gestão da saúde humana e do ecossistema, integram as reflexões finais.

\section{A saúde ambiental e a construção de um novo campo científico}

A relação entre o meio ambiente e a saúde humana é discutida há bastante tempo. Registros são encontrados em obras de grandes filósofos da humanidade9. Contudo, ao longo da história, esta associação nem sempre foi pacífica. O método científico mecanicista do século XVII colocou a fragmentação e o reducionismo como pressuposto básico da produção do conhecimento. A biologia, que se originou a partir deste arcabouço, ao adotar a noção isolada de corpo, influenciou a medicina ${ }^{10}$. Esta perspectiva distanciou a saúde da temática do meio ambiente.

Em termos conceituais, até a década de 1940, os estudos sobre essa relação restringiam-se às questões relativas à água potável e ao saneamento básico. Somente quando a Organização Mundial da Saúde (OMS) formulou seu conceito original de saúde, considerando que "a saúde é o estado de completo bem-estar físico, mental e social, não sendo apenas a mera ausência de doença ou enfermidade" ${ }^{11}$, resgatou-se a concepção integral de saúde.

Na segunda metade do século XX, no bojo do debate ambiental internacional, ocorre uma diferenciação nos enfoques dos problemas ambientais relacionados à saúde humana. Surgem, então, duas vertentes. A primeira, a "verde", preocupa-se com os efeitos da atividade humana sobre o meio ambiente, o desenvolvimento sustentável, a dinâmica demográfica, a destruição da camada de ozônio, o desmatamento, dentre outros. A ver- 
tente "azul", por sua vez, toma como referência os efeitos do ambiente sobre a saúde e o bem-estar da humanidade, ou seja, a saúde ambiental ${ }^{12}$.

Esta perspectiva desenvolve e aporta um novo e ampliado conceito de saúde ambiental, integrando os fatores determinantes da saúde humana e do ecossistema ${ }^{1}$. Neste contexto, em 1972, a OMS chama a atenção para o controle dos processos químicos, físicos e biológicos como influências e fatores que exerceriam efeitos sobre o indivíduo ou a sociedade ${ }^{13}$. Em 1989, o conceito é revisado e dividido em duas partes: uma teórica, voltada para o conhecimento dos aspectos da saúde determinados pelo meio ambiente, e outra, prática, que amplia o conceito de avaliação, correção, controle e prevenção dos fatores ambientais que podem afetar de forma adversa a saúde da presente e das futuras gerações ${ }^{1}$.

Em decorrência dessa nova interpretação, vários enfoques foram propostos para o entendimento das complexas relações entre os ambientes e os padrões de saúde. Nas Américas, uma referência importante foi o Modelo Lalonde, adotado pelo governo canadense em 1974, na reorganização da área de saúde pública ${ }^{14}$. A sua institucionalização se deu por meio da Carta de Ottawa, de $1986^{15}$. Os fatores ambientais, tanto quanto os hábitos de vida, passam a ser reconhecidos como determinantes, integrantes ou componentes da saúde do indivíduo ${ }^{2,5,8}$.

A saúde ambiental incorpora, por conseguinte, questões como: poluição química, pobreza, equidade, condições psicossociais e os pressupostos do desenvolvimento sustentável. Neste cenário, um novo leque de pesquisas científicas se abre. São incorporados estudos de poluentes químicos ambientais, causadores de doenças; a pesquisa epidemiológica, relacionada a alterações terrestres e do ecossistema aquático e a mudanças climáticas; estudos relacionando a pobreza às alterações no perfil de morbimortalidade ${ }^{16}$. Além disso, passam a compor a agenda de pesquisa aspectos como os efeitos da mudança climática sobre a sustentabilidade do ecossistema e a saúde humana; a interação entre ambiente, desenvolvimento e saúde humana; e a gestão dessas mudanças nas escalas local, regional e global ${ }^{17}$.

No âmbito da discussão, as incertezas e a complexidade inerentes aos sistemas socioecológicos passam a conformar os principais desafios no que se refere à pesquisa em saúde ambiental. Fatores que assinalam as dificuldades de se criar e adaptar métodos e práticas que cruzam as fronteiras disciplinares, valorizando os saberes não científicos.

\section{A geração inter e trans-disciplinar do conhecimento em saúde ambiental}

Se a compreensão da matriz de determinantes da saúde, em sua complexidade, requer o diálogo entre os diferentes campos do saber, a questão central repousa em dois pontos: (i) abrir as fronteiras entre as disciplinas, para reintegrar o conhecimento $^{18,19}$, e (ii) cruzar as fronteiras da ciência, para ir ao encontro do mundo da vida ${ }^{3}$. Isso amplia não somente a capacidade de conhecer, mas também de intervir ${ }^{20}$.

Neste sentido, inter e transdisciplinaridade figuram como tentativas de troca e cooperação entre os campos científicos e os saberes não científicos. Entretanto, em termos de definições, o que se encontra na literatura é bastante variado e diverso $^{17}$. São enfoques que, por vezes, figuram como sinônimos, por outras, se confundem e contradizem, por serem difíceis de definir, polissêmicos e imprecisos ${ }^{21}$.

No entanto, durante a última década, despontaram alguns esforços importantes para conceituar as diferentes dimensões da interação entre disciplinas, sobretudo no que tange à inter e transdisciplinaridade. As principais contribuições incluem Janstsch ${ }^{22}$, Gibbons ${ }^{23}$, Habermas ${ }^{24}$, Klein ${ }^{25}$ e Morin ${ }^{26}$.

No que se refere à saúde ambiental, importantes estudos sobre os desafios da inter e transdisciplinaridade foram publicados na última década. Dentre as principais contribuições destacam-se: Forget e Lebel ${ }^{27}$, Lebel ${ }^{5}$, Nielsen ${ }^{28}$ e Waltner-Toews et al. ${ }^{29}$. No caso brasileiro, têm se destacado os estudos desenvolvidos por pesquisadores da Fundação Oswaldo Cruz (Fiocruz) ${ }^{3,8,30}$.

Embora se encontre uma pluralidade de definições em torno da inter e da transdisciplinaridade $^{31}$, algumas convergências podem ser notadas. No campo da saúde ambiental, por exemplo, o tipo de colaboração que se estabelece entre os agentes que participam da geração de conhecimento tem sido usado na distinção entre a inter e a transdisciplinaridade. Assim, se a colaboração baseia-se na busca por uma solução puramente científica e contribuem apenas cientistas, o processo tem sido reconhecido como interdisciplinar ${ }^{4}$. É uma forma específica de pesquisa que se configura pela integração de dados, métodos, ferramentas, conceitos e teorias de diferentes disciplinas para criar uma visão integral e entender questões complexas ${ }^{32,33}$.

Adicionalmente, se essa colaboração interdisciplinar integra os indivíduos que representam o grupo de pessoas afetadas, caracteriza-se um 
processo transdisciplinar ${ }^{3,5}$. A marca desse enfoque são as conexões que se estabelecem entre a pesquisa acadêmica e a esfera do saber informal e contextual e popular ${ }^{34,35}$. A participação dos atores locais no processo de pesquisa é o elemento fundamental. Nesta perspectiva, parte do pressuposto de que a solução dos problemas de saúde ambiental depende não somente do uso da técnica, mas principalmente da análise e intervenção no que tange à compreensão e à resolução de conflitos ${ }^{4,17,35}$.

Estas novas estratégias de produzir conhecimento distinguem-se consideravelmente das tradicionais pesquisas acadêmicas. Como conseguirá, então, o pesquisador adaptar-se a esta nova dinâmica? Em que medida é possível constituir grupos de pesquisa que cruzem as fronteiras das disciplinas e dialoguem com saberes não científicos? Trata-se de desafios que se apresentam em duas dimensões: individual e coletiva ${ }^{33,36}$, pois se entende que a integração do conhecimento pode ocorrer dentro de uma única mente, assim como entre membros de uma equipe. Nesta perspectiva, a literatura tem abordado aspectos individuais, como expectativas, metas e experiências, e aspectos coletivos, como a composição da equipe, gestão de projetos e medidas de eficácia ${ }^{33}$.

\section{Pesquisa individual e em equipes: entre desafios, oportunidades e limitações}

As barreiras conceituais, sociais e institucionais que desafiam a prática da inter e transdisciplinaridade na pesquisa científica têm sido tema de diversas conferências e seminários acadêmicos ${ }^{31,35}$. Sem a pretensão de esgotar o assunto, propomonos a contribuir para esse o debate. Para tanto, integramos referenciais teóricos das ciências naturais e das ciências sociais, buscando abordar sob uma nova perspectiva alguns dos principais desafios que a saúde ambiental coloca ao pesquisador individual e à prática da pesquisa coletiva.

\section{Uma âncora científica}

A inter e a transdisciplinaridade, ao mesmo tempo em que priorizam a integração entre diferentes disciplinas, valorizam o trabalho especializado. O papel do pesquisador é, em primeiro lugar, alimentar a conexão que se estabelece entre as disciplinas, por meio de conhecimentos científicos e técnicos trazidos de sua área ${ }^{19}$. Contrariamente, se o pesquisador da saúde ambiental secundariza suas especialidades em detrimento do conhecimento menos especializado, o resultado final pode ser apenas uma ponte genérica entre o natural e o social, ao invés da unidade do conhecimento $^{37}$.

\section{Conhecer o desconhecido}

A abertura ao novo é essencial, principalmente diante das diferentes atitudes, hábitos e costumes que caracterizam a cultura de cada campo científico. Este desafio requer a apreensão da relevância e a aplicação de diferentes linguagens, conceitos, conteúdos e métodos que concernem às disciplinas científicas ${ }^{36}$. Além disso, a intervenção no plano da saúde ambiental requer disposição para lidar com conhecimentos técnicos (planejamento, facilitação, cogestão, supervisão, avaliação, etc. $)^{2,38}$ e com conflitos sociais e estratégias políticas ${ }^{30}$.

\section{Dialogar e articular}

O diálogo entre as disciplinas requer o estabelecimento de princípios epistemológicos e metodológicos, não para a criação de uma metalinguagem interdisciplinar, o que fragilizaria os corpos teóricos das diferentes áreas, mas sim para permitir um grau de concordância entre os diferentes campos disciplinares, articulando os conhecimentos científicos e técnicos ${ }^{19,39}$. No campo da intervenção, por sua vez, as dificuldades de comunicação demandam a superação do formalismo excessivo, da rigidez das definições e do absolutismo da objetividade ${ }^{40}$. Para tanto, a parceria com a comunidade tem de ser suficientemente flexível. Isso exige esforço, de ambas as partes, na ampliação do diálogo e na colaboração dinâmica ${ }^{38}$.

\section{Aumentar a credibilidade}

Em raros casos, a ciência valorizou a dinâmica de aprendizagem que se estabelece entre o pesquisador e o pesquisado ${ }^{41}$. Esse desafio impõe novas formas de pensar a credibilidade em diferentes níveis: acadêmico, comunitário e político. Em um contexto geral, duas estratégias têm sido empregadas na busca por credibilidade juntos aos pares, no campo da saúde ambiental: na primeira, os esforços têm convergido para a criação de indicadores baseados em uma visão integral do ambiente ${ }^{42}$. Trata-se de desenvolver indicadores, fazer modelagens e construir cenários futuros, baseados em leis estáveis universais, nos quais os sistemas sociais, tecnológicos e ecológicos interagem sem historicidade ${ }^{43}$. 
Em outra estratégia pretende-se integrar a historicidade e a espacialidade dos fenômenos ${ }^{40}$. Mobiliza-se, para tanto, uma abordagem baseada na teoria dos sistemas auto-organizados, holárquicos e abertos ( $\mathrm{SOHO}$ ), pretendendo lidar com múltiplas escalas e perspectivas e elevados graus de incerteza. Trata-se de um método que inclui modelos, diagramas, mapas e representações, integrando o conhecimento local à pesquisa por meio de métodos antropológicos e sistemas geográficos ${ }^{44,45}$. Em ambas as vertentes, tem se discutido a necessidade de ampliar a capacidade de investigação, publicação e divulgação ${ }^{46}$.

\section{Abordagem ecossistêmica: ferramenta para a inter e a transdisciplinaridade}

Se a saúde ambiental pretende se apoiar em abordagens inter e transdisciplinares para integrar diferentes disciplinas e produzir resultados concretos, então ela necessita de um enfoque teórico e metodológico que a subsidie. É nesta perspectiva que nas últimas décadas a abordagem ecossistêmica para a saúde humana (ecosaúde) vem ganhando proeminência em países e regiões como o Canadá, América Latina, Caribe, Oriente Médio e a África ${ }^{47,48}$. Ela se propõe a conectar a gestão ambiental com uma compreensão abrangente da saúde humana, incluindo fatores sociais, econômicos e culturais inerentes a um dado ecossistema ${ }^{46}$.

As primeiras formulações da ecosaúde surgiram ainda na década de 1970, a partir de um estudo realizado na região dos Grandes Lagos, um ecossistema compartilhado pelo Canadá e os Estados Unidos ${ }^{49}$. Na ocasião, a complexidade dos processos predatórios na bacia hidrográfica chamou a atenção de um grupo de pesquisadores da Comissão Mista Internacional dos Grandes Lagos, do Canadá. Duas questões teóricometodológicas instigaram a equipe: (i) a percepção da insuficiência teórica de abordagens unidisciplinares para compreender as complexas interações entre os sistemas social e ecológico; (ii) a necessidade do envolvimento das autoridades, dos gestores, das empresas e dos cidadãos para obter êxito nas propostas ${ }^{49}$. Os esforços culminaram na construção de um enfoque que garantisse a integridade do sistema e a continuidade do desenvolvimento local ${ }^{27}$.

Desde então, essa abordagem vem se consolidando por intermédio de programas de pesquisa e linhas de financiamento como uma estratégia para a integração transdisciplinar em saúde ambiental ${ }^{27,48,50}$. Ao longo do processo, surgiram programas específicos de pesquisa, como Ecohealth, do Centro Internacional de Investigações para o Desenvolvimento (IDRC) do Canadá e o Ecosalud, da Organização Pan-Americana da Saúde (OPAS) ${ }^{5,51}$. Constituíram-se equipes de pesquisa em diversos países e diferentes continentes. Criou-se uma tradição de pesquisa em torno da relação entre questões como saúde e agricultura, poluição ambiental, urbanização, doenças transmissíveis e implicações para a saúde decorrentes das mudanças climáticas ${ }^{48,52,53}$.

Ao longo dessas quatro décadas de experiência, as bases teóricas e metodológicas das dinâmicas de pesquisa e intervenção evoluíram e sofreram adaptações. Neste processo, duas vertentes se distinguiram. A primeira centra-se na identificação e mensuração de aspectos que provocam alterações nos ecossistemas e têm potencial para afetar a saúde humana. Baseia-se em métodos e técnicas que fornecem informações científicas para a tomada de decisão e gestão do ecossistema $^{54}$. Para a operacionalização, incorpora a construção de indicadores que ajudam no diagnóstico das causas da disfunção dos ecossistemas e na análise da potencial solução, reduzindo os riscos de uma patologia. Como metodologia, incorpora o mapeamento da estrutura dos ecossistemas e a modelagem de suas funções. Para tanto, faz uso de tecnologias de informação para compreender as suas relações funcionais, na busca por metas de gestão ${ }^{55,56}$.

A segunda vertente, por sua vez, valoriza o desenvolvimento de uma abordagem contextualizada e participativa para compreender e buscar as estratégias de gestão em saúde de comunidades locais (aldeias, vilas e pequenas cidades, por exemplo $)^{54}$. Busca valorizar a integração de todos os atores, interessados e responsáveis, na construção do conhecimento e na solução dos problemas ${ }^{3,5,27}$. O objetivo central são as mudanças em termos de valores sociais. Estes estão relacionados com aquilo que define o que é saudável para as pessoas que vivem nessas comunidades ${ }^{50,57}$.

Em ambas as perspectivas, a ideia de mudança é fundamental. Ela depende da integração das ciências naturais e sociais com os valores humanos (interdisciplinaridade), da participação do conjunto de atores envolvidos (transdisciplinaridade), e de um terceiro pilar metodológico da ecosaúde, a equidade social e de gênero ${ }^{3,5,27,56}$. Estas são consideradas variáveis políticas e socioeconômicas por meio das quais se pode analisar papéis, responsabilidades, contradições e dificuldades ${ }^{3}$.

Em termos de experiência prática, os resultados de algumas iniciativas nos ajudam a perceber 
em que medida a inter e a transdisciplinaridade podem influenciar os resultados das pesquisas e subsidiar a intervenção. Neste sentido, revisamos dois exemplos: o projeto brasileiro denominado CARUSO (Contaminação e exposição ao mercúrio na Amazônia) $)^{5,58}$ e um conjunto de projetos desenvolvido no Nepal (transmissão urbana de equinococose em Katmandu) $)^{59,60}$.

\section{Projeto Caruso}

Os resultados do projeto Caruso permitem ilustrar duas questões em particular: (i) a insuficiência do enfoque unidisciplinar ${ }^{27}$; e (ii) a importância da participação dos diversos grupos sociais $^{58,61}$. Trata-se de uma experiência financiada pelo IDRC que agrega, desde 1994, pesquisadores brasileiros e canadenses na caracterização das fontes, das vias de transmissão e dos efeitos do mercúrio sobre a saúde das comunidades na região do médio Tapajós, Estado do Pará, Bra$\mathrm{sil}^{62}$. Inclui, igualmente, o desenvolvimento de estratégias colaborativas para reduzir as fontes e a exposição ao mercúrio ${ }^{58}$.

Para compreender como a inserção da pesquisa transdisciplinar provocou mudanças na elaboração de noções científicas, é importante revisar alguns aspectos históricos da geração de conhecimento sobre o mercúrio na Amazônia. As pesquisas realizadas desde os anos 1970 indicavam que as altas concentrações de mercúrio, encontradas em peixes carnívoros e no cabelo de moradores ribeirinhos, derivavam da atividade de mineração de ouro. Tratava-se de estudos focados nos aspectos biológicos, geoquímicos ou médicos do problema ${ }^{63}$. Os seus resultados eram plenamente aceitos pela comunidade científica.

Quando, porém, uma equipe formada por especialistas de pesca, biogeoquímica, ecologia aquática, toxicologia, agricultura, saúde humana, comunicação, antropologia e nutrição, analisou a exposição ao mercúrio na bacia do Tapajós, veio à tona uma matriz bem mais complexa de fatores que provocam a perturbação da saúde humana e dos ecossistemas ${ }^{5,27,58,64}$. Percebeu-se que, além da ação dos garimpos de ouro, a contaminação do solo, dos peixes e da população está associada à mobilização do mercúrio naturalmente presente nos solos e ecossistemas aquáticos. Essa mobilização é provocada pelo desmatamento e potencializada pela erosão e lixiviação do solo, geradas pela tradicional prática agrícola amazônica de corte e queima ${ }^{63,65}$.

A integração entre os conhecimentos científicos e os saberes dos moradores sobre os ecossis- temas locais contribuiu para a elaboração de respostas à problemática do mercúrio e desencadeou mudanças ${ }^{58}$. A pesquisa científica sobre a concentração de mercúrio em peixes mostrou que a concentração variava em espécies piscívoras e herbívoras ${ }^{66}$. O diálogo entre pesquisadores e comunidades viabilizou a transformação destes resultados de pesquisa em conhecimentos para a ação. A partir de reuniões com mulheres e homens, agricultores, professores, agentes de saúde e autoridades locais, desenvolveu-se uma campanha baseada no lema: "Comer mais peixes que não comem outros peixes" 67 . Assim, em sistema de colaboração, iniciou-se um processo de adaptação da dieta alimentar e localização de novas seções do rio para a atividade de pesca. Reorientando o consumo de peixe para as espécies menos contaminadas, as comunidades puderam minimizar o risco tóxico associado à exposição ao mercúrio, sem deixar de consumir este componente essencial para a dieta ribeirinha ${ }^{68}$. Além disso, o envolvimento das mulheres no levantamento da dieta das comunidades ribeirinhas permitiu identificar uma relação entre o consumo regular de frutas e uma diminuição dos níveis de mercúrio no sangue ${ }^{62}$. A hipótese dos pesquisadores é de que o consumo de frutas pode influenciar a absorção de mercúrio ${ }^{69}$. A descoberta abriu mais uma perspectiva de ação para diminuir a exposição a este produto.

Essa experiência aponta como por meio da transdisciplinaridade se chegou à compreensão integral da dinâmica local do mercúrio no ambiente $^{64}$ e o desenvolvimento de soluções viáveis para um problema complexo. Atualmente a comunidade científica reconhece as transformações socioecológicas ocorridas nas comunidades ${ }^{58}$, em termos de redução do impacto ambiental e social da contaminação por mercúrio, como resultado de uma combinação de engajamento local e investigação científica ${ }^{60}$.

\section{Katmandu, Nepal}

No mesmo sentido dos resultados do Projeto Caruso, uma iniciativa desenvolvida no Nepal revela que um problema, por vezes considerado simples, quando analisado a partir das visões de diferentes disciplinas e com a participação das pessoas envolvidas, expõe sua complexidade ${ }^{60}$.

Desde a década de 1970, pesquisadores do Centro Nacional de Pesquisa de Zoonoses e Higiene Alimentar, da Universidade de Guelph, do Canadá, e do Centro de Controle e Prevenção Doenças de Atlanta e a Universidade de Salford, 
da Inglaterra, vem desenvolvendo estudos sobre a exposição humana ao parasita Echinococcus granulosus, agente da equinococose canina e humana. Três fases marcam os projetos de pesquisa: Fase 1: abordagem epidemiológica (19921996); Fase 2: abordagem ecossistêmica (19982001); Fase 3: o significado dos modelos: a metanarrativa (a partir de 2001) ${ }^{59}$. As duas primeiras ilustram o enfoque que pretendemos descrever.

Na primeira fase, uma série de estudos epidemiológicos determinou as taxas de infecção em animais e humanos e identificou os fatores de risco da exposição humana ao parasita ${ }^{59}$. Os resultados indicaram a necessidade de inspecionar, tratar e controlar os cães e a carne de animais destinados à alimentação, conscientizar a população e criar um programa de vigilância epidemiológica $^{70}$. Essas ações foram implementadas após a etapa de investigação e alcançaram algum sucesso na prevenção da doença. Contudo, as rápidas mudanças sociais e ecológicas colocaram muitas conquistas em cheque ${ }^{45}$. Como sugestões, os pesquisadores propuseram um modelo sistêmico, participativo e integrativo de pesquisa, reconhecendo que o contexto ambiental e social de produção da doença era bem mais complexo do que o esperado ${ }^{59,71}$. Um quadro de mudanças exigia a participação da sociedade na vigilância epidemiológica, em campanhas de saúde pública e no desenvolvimento da comunidade local ${ }^{72}$.

$\mathrm{Na}$ segunda fase do projeto, os estudos epidemiológicos deram lugar a uma abordagem ecossistêmica desenvolvida por meio de práticas transdisciplinares e participativas ${ }^{59,73}$. O resultado mostrou porque as ações preventivas não corriam como o esperado. A sociedade nepalense apresenta uma configuração bastante complexa. A estratificação por um sistema de castas determina os locais de moradia e as profissões. Estas, por sua vez, estão relacionadas com a disseminação da doença, os índices de morbidade e a expectativa de vida ${ }^{74}$. Para reduzir a prevalência da doença na população, foi necessário o esforço dos pesquisadores para a compreensão das interações entre os determinantes socioculturais, políticos, econômicos e ambientais da saúde humana, o engajamento da comunidade, a capacitação dos indivíduos e grupos locais com foco na criação de mais ambientes saudáveis ${ }^{72}$.

Desafiados por essa realidade, os pesquisadores construíram uma metodologia adaptativa para a sustentabilidade dos ecossistemas e saúde (AMESH), baseada na teoria dos sistemas autoorganizados, holárquicos e abertos (SOHO). Trata-se da construção colaborativa de diagra- mas de fluxo que orientam o planejamento e a ação, complementados com métodos qualitativos, tais como a pesquisa-ação participante (PAP) e a pedagogia de Paulo Freire ${ }^{44,59,75}$. Os diagramas contribuíram para descrever o ciclo do parasita, identificar interações dos grupos comunitários, bem como as áreas de potencial conflito. Os resultados permitiram a definição dos pontos de intervenção.

O projeto levou o governo nepalês a elaborar e aprovar uma nova lei sobre o abate de animais e inspeção da carne. Por meio de compostagem, os açougueiros transformaram os resíduos dos animais em fertilizantes. Além disso, houve melhorias na coleta e reciclagem do lixo, nos sistemas de esgoto, banheiros públicos e instalações sanitárias ${ }^{71,74}$.

Diante da melhor compreensão das diferenças culturais relacionadas com as castas, grupos de atores de duas comunidades vizinhas de grupos distintos foram chamados a participar do projeto, juntamente com uma organização não governamental local ${ }^{59}$. As estratégias participativas e transdisciplinares resultaram em uma maior capacidade organizativa para resolver as questões locais.

\section{Considerações finais}

À medida que a ciência vai identificando suas limitações, surgem novos enfoques analíticos e metodológicos com o propósito de aumentar o alcance das análises, aproximando cada vez mais os resultados das pesquisas à complexidade que se manifesta nos fenômenos sociais e ambientais. Em termos gerais, a saúde ambiental oferece esta perspectiva. Em primeiro lugar porque se desdobra sobre a integração do conhecimento das ciências naturais e humanidades. Em segundo, porque se lança no desafio de incorporar e valorizar os saberes locais. A mútua influência entre estas perspectivas permite avançar na pesquisa e na participação da gestão da saúde humana e do ecossistema.

Oportunidades e limitações são intrínsecas a esse processo. Por um lado, trata-se de romper com as fronteiras historicamente construídas entre as disciplinas, buscando integrar dados, métodos, ferramentas, conceitos e teorias. Por outro, incorporar o saber informal e a participação social e lidar com relações de poder e conflitos, no caminho do estabelecimento de vínculos entre o conhecimento do problema e a sua resolução.

$\mathrm{Na}$ prática do dia-a-dia, essa nova proposta exige a composição de equipes de trabalho. Nes- 
sa dinâmica, afloram aspectos individuais, expectativas, metas e experiências que podem limitar ou potencializar a eficácia dos métodos e resultados. O pesquisador se vê diante de uma estreita margem entre valorizar sua especialidade, ao mesmo tempo em que cede a outras linguagens, conceitos, métodos, conhecimentos. A reflexão e a autocrítica se tomam relevância nesse processo, uma vez que a postura individual, flexibilidade, abertura à subjetividade, ao diálogo $\mathrm{e}$ à colaboração dinâmica, influenciam de forma substantiva as atividades dos grupos.

Em termos de credibilidade científica, revelase uma dialética entre criar indicadores, modelagens e desenvolver cenários, aceitos pelos pares, porém sem historicidade, ou desenvolver modelos, diagramas, mapas e representações subjetivos, históricos e políticos, mas que exigem o es- forço de ilustrar de maneira convincente seus méritos científicos. Seja qual for a opção, a saúde ambiental, como outras ciências interdisciplinares, se vê diante do imperativo de ampliar sua capacidade de investigação, publicação e divulgação e conquistar um espaço maior na ciência.

A opção pela ecossaúde é uma das alternativas viáveis para a ampliação desse espaço. Os projetos desenvolvidos na região dos Grandes Lagos, no Canadá, na Amazônia brasileira ou em Katmandu, no Nepal, demonstram esse potencial. Os esforços pela superação da insuficiência teórica das abordagens unidisciplinares e em prol da participação de atores locais, indicam a possibilidade concreta de contribuir para a resolução de questões locais, ao tempo que se exploram novas teorias e modelos científicos, investindo na geração de conhecimentos.

\section{Colaboradores}

M Weihs e F Mertens contribuíram igualmente na elaboração do artigo, desde a concepção à redação final.

\section{Referências}

1. Rengifo Cueller H. Conceptualización de la salud ambiental: teoría y práctica (parte 1). Rev Perú Med Exp Salud Publica 2008; 25(4):403-409.

2. Rengifo Cueller H. Conceptualización de la salud ambiental: teoría y práctica (parte 2). Rev Perú Med Exp Salud Publica 2009; 26(1):66-73.

3. Gómez CM, Minayo MCS. Enfoque ecossistêmico de saúde: uma estratégia transdisciplinar. InterfacEHS 2006; 1(1):1-19.

4. Balsiger P. Supradisciplinary research practices: history, objectives and rationale. Futures. 2004; 36(4):407-421.

5. Lebel J. Health: an ecosystem approach. Ottawa: International development research centre; 2003.

6. Pohl C. From science to policy through transdisciplinary research. Environ Sci Policy 2008; 11(1):46-53.

7. Aguirre A, Wilcox BA. EcoHealth: envisioning and creating a truly global transdiscipline. EcoHealth 2008; 5(3):238-239.

8. Minayo M. Enfoque ecossistêmico de saúde e qualidade de vida. In: Minayo M, Miranda AC, organizadores. Saúde e ambiente sustentável: estreitando nós. Rio de Janeiro: Fiocruz; 2002. p.173-190.

9. Gouveia N. Saúde e meio ambiente nas cidades: Os desafios da saúde ambiental. Saúde Soc. 1999; 8(1): 49-61.

10. Czeresnia D. Interfaces do corpo: integração da alteridade no conceito de doença. Rev bras epidemiol 2007; 10(1):19-22.

11. World Health Organization (WHO). Commission on Health and Environment. Preamble to the constitution of the World Health Organization as adopted by the International Health Conference. Geneve: WHO; 1946. 
12. Ordóñez GA. Salud ambiental: conceptos y actividades. Rev Panam Salud Publica 2000; 7(3):137-147.

13. World Health Organization (WHO). Commission on Health and Environment. Nuestro planeta, nuestra salud: informe de la Comisioin de Salud y Medio Ambiente de la Organización Mundial de la Salud. Washington D.C.: Organizacioìn Panamericana de la Salud; 1993.

14. Gouvernement du Canada. Nouvelle perspective de la santé des Canadiens: un document de travail [Santé national et du Bien-être social, 1981]; 2009.

15. World Health Organization (WHO). Ottawa charter for health promotion. First International Conference on Health Promotion. Geneve: WHO; 1986.

16. Camara VM, Tambellini AT. Considerações sobre o uso da epidemiologia nos estudos em saúde ambiental. Rev bras epidemiol 2003; 6(2):95-104.

17. Wilcox B, Kueffer C. Transdisciplinarity in ecoHealth: status and future prospects. EcoHealth 2008; $5(1): 1-3$.

18. Leff E. Complexidade, interdisciplinaridade e saber ambiental. In: Philippi-Junior A, Tucci CEM, Hogan DJ, Navegantes R, organizadores. Interdisciplinaridade em ciências ambientais. São Paulo: Signus Editora; 2000. p. 19-51.

19. Leff E. Epistemologia Ambiental. 3a Edição. São Paulo: Ed. Cortez; 2002.

20. Erdmann AL, Schlindwein BH, Sousa FGM. A produção do conhecimento: diálogo entre os diferentes saberes. Rev Bras Enf 2006; 59(4):560-564.

21. Morin E. A cabeça bem feita: repensar a reforma, reformar o pensamento. 16a Edição. Rio de Janeiro: Bertrand Brasil; 2009.

22. Jantsch E. Forecasting and the systems approach: a critical survey. Policy Sci 1972; 3(4):475-498.

23. Gibbons M. Transfer sciences: management of distributed knowledge production. Empirica 1994; 21(3):259-270.

24. Habermas J. Knowledge and human interests. Boston: Beacon Press; 1971.

25. Klein J. Interdisciplinarity: history, theory, and practice. Detroit: Wayne State University Press; 1990.

26. Morin E. Ciência com consciência. $4^{\text {a }}$ Edição. Rio de Janeiro: Bertrand Brasil; 2000.

27. Forget G, Lebel J. An ecosystem approach to human health. Int J Occup Med Environ Health 2001; 7(2):3-38.

28. Nielsen NO. Ecosystem approaches to human health. Cad Saude Publica 2001; 17(Supl.):569-575.

29. Waltner-Toews D, Kay JJ, Lister NME. The ecosystem approach: complexity, uncertainty, and managing for sustainability. New York: Columbia University Press; 2008.

30. Freitas CM. As ciências sociais e o enfoque ecossistêmico de saúde. In: Minayo MCS, Coimbra Júnior CEA, organizadores. Críticas e atuantes: ciências sociais e humanas em saúde na América Latina. Rio de Janeiro: Fiocruz; 2005. p. 47-59.

31. Lawrence RJ, Despres C. Futures of Transdisciplinarity. Futures 2004; 36(4):397-405.

32. Jacobs JA, Frickel S. Interdisciplinarity: a critical assessment. Annu Rev Sociol 2009; 35:43-65.

33. Wagner CS, Roessner JD, Bobb K, Klein JT, Boyak KW, Keyton J, Rafols I, Börner K. Approaches to understanding and measuring interdisciplinary scientific research (IDR): A review of the literature. $J$ Informetrics 2011; 5(1):14-26.
34. Stokols D, Fuqua J, Gress J, Harvey R, Phillips K, Baezconde-Garbanati L, Unger J, Palmer P, Clark MA, Colby SM, Morgan G, Trochim W. Evaluating transdisciplinary science. Nicotine \& Tobacco Res 2003; 5(Supl. 1):S21-S39.

35. Horlick-Jones T, Sime J. Living on the border: knowledge, risk and transdisciplinarity. Futures 2004; 36(4):441-456.

36. Lynch J. It's not easy being interdisciplinary. Int $J$ Epidemiol 2006; 35(5):1119-1122.

37. Nicolescu B. Um novo tipo de conhecimento: transdisciplinaridade. In: Nicolescu B, Pineau G, Maturana $\mathrm{H}$, Random M, Taylor P, organizadores. Educação e transdisciplinaridade. Brasília: UNESCO; 2000. p. 13-29.

38. Bopp M. Transdisciplinarity and participation: an evaluation of transdisciplinarity and participatory aspects of the IDRC ecosystem approaches to human health project initiative. Final report. Canadá: Four Worlds Centre for Development Learning; 2001.

39. Almeida-Filho N. Complejidad y transdisciplinariedad en el campo de la salud colectiva: evaluación de conceptos y aplicaciones. Salud Colectiva 2006; 2(2):123-146.

40. Freitas L, Morin E, Nicolescu B. Carta da transdisciplinaridade. Convento de Arrábida: Primeiro Congresso Mundial da Transdisciplinaridade; 1994.

41. Thiollent M. Pesquisa-ação nas organizações. São Paulo: Atlas; 1997.

42. Forget G. Un mejor manejo del ecosistema para una mejor salud humana: El enfoque ecosistémico para la salud humana. In: Feola G, Bazzani R, organizadores. Desafíos y estrategias para a implementación de un enfoque ecossistémico para la salud humana en los países em desarollo - reflexiones a propósito de las consultas regionales. Montevideo: Centro Internacional de Investigaciones para el Desarrollo; 2002. p. 57-65.

43. Porto MFS. Uma ecologia política dos riscos: princípios para integrarmos o local e o global na promoção da saúde e da justiça ambiental. Rio de Janeiro: Fiocruz; 2007.

44. Waltner-Toews D, Kay JJ, Neudoerffer C, Gitau T. Perspective changes everything: managing ecosystems from the inside out. Fron Ecol Environ 2003; 1:23-30.

45. Neudoerffer RC, Waltner-Toews D, Kay JJ, Joshi DD, Tamang MS. A diagrammatic approach to understanding complex eco-social interactions in Kathmandu, Nepal. Ecol and Soc 2005; 10(2):12.

46. Feola G, Bazzani R. Desafíos y estrategias para a implementación de un enfoque ecossistémico para la salud humana en los países em desarollo - reflexiones a propósito de las consultas regionales. Montevideo: Centro Internaciónal de Investigaciones para el Desarrollo; 2002.

47. Parkes MW, Charron D, Sánchez A. Better together: Field-building networks at the frontiers of ecohealth research. In: Charron D, editors. Ecohealth research in practice: innovative applications of an ecosystem approach to health. Ottawa: International Development Research Centre; 2012. p. 231-254.

48. Webb JC, Mergler D, Parkes MW, Saint-Charles J, Spiegel J, Waltner-Toews D, Yassi A, Woollard. Tools for thoughtful action: the role of ecosystem approaches to health in enhancing public health. Can J Public Health 2010; 101(6):439-441. 
49. Great Lakes Research Advisory Board. The Ecosystem Approach: Scope and implications of an ecosystem approach to transboundary problems in the Great Lakes Basin. Windsor: Special Report to the IJC; 1978.

50. Minayo MCS. Contribuições da antropologia para pensar a saúde. In: Campos G, organizador. Tratado de saúde coletiva. Rio de Janeiro: Ed. Fiocruz; 2009. p. 189-218.

51. Augusto LGS, Brasil MGF, Franco-Netto G. Abordagens integradas para vigilância em saúde ambiental. In: Miranda AC, Barcellos C, Moreira JC, Monken M, organizadores. Território, ambiente e saúde. Rio de Janeiro: Fiocruz; 2008. p. 183-203.

52. Boischio A, Sánchez A, Orosz Z, Charron D. Health and sustainable development: challenges and opportunities of ecosystem approaches in the prevention and control of dengue and Chagas disease. Cad Saude Publica 2009; 25(Supl. 1):S149-S154.

53. Charron D. Ecohealth Research in Practice. In: Charron D, editors. Ecohealth research in practice: innovative applications of an ecosystem approach to health. Ottawa: International Development Research Centre; 2012. p. 255-271.

54. Freitas CM, Oliveira SG, Schütz GE, Freitas MB, Camponovo MPG. Ecosystem approaches and health in Latin America. Cad Saude Publica 2007; 23(2):283-296.

55. VanLeeuwen JA, Waltner-Toews D, Abernathy T, Smitt B. Evolving models of human health toward an ecosystem context. Ecosystem Health 1999; 5(3):204-219.

56. Nielsen ON. Enfoques ecosistémicos para la salud humana. In: Feola G, Bazzani R, organizadores. Desafíos y estrategias para a implementación de un enfoque ecossistémico para la salud humana en los países em desarollo - reflexiones a propósito de las consultas regionales. Montevideo: Centro Internacional de Investigaciones para el Desarrollo; 2002. p. 9-16.

57. Rapport DJ, Constanza R, McMichael AJ. Assessing ecosystem health. Tree 1998; 13(10):397-402.

58. Mertens F, Saint-Charles J, Mergler D, Passos CJ, Lucotte M. A network approach for analysing and promoting equity in participatory Ecohealth research. Ecohealth 2005; 2:116-123.

59. Waltner-Toews D, Neudoerffer C, Joshi DD, Tamang MS. Agro-urban ecosystem health assessment in Kathmandu, Nepal: epidemiology, systems, narratives. EcoHealth 2005; 2:155-164.

60. Parkes MW, Bienen L, Breilh J, Hsu L-N, McDonald M, Patz JA, Rosenthal JP, Sahani M, Sleigh A, Waltner-Toews D, Yassi A. All hands on deck: transdisciplinary approaches to emerging infectious disease. EcoHealth 2005; 2:258-272.

61. Mertens F, Saint-Charles J, Mergler D, Lucotte M. Emergence and robustness of a community discussion network on mercury contamination and health in the Brazilian Amazon. Health Educ Behav 2008; 35(4):509-521.

62. Guimarães JRD, Mergler D. A virtuous cycle in the Amazon: reducing mercury exposure from fish consumption requires sustainable agriculture. In: Charron D, organizador. Ecohealth Research in Practice: innovative Applications of an Ecosystem Approach to Health. Ottawa: International Development Research Centre; 2012. p. 109-118.
63. Guimarães JR. Origins and effects of mercury on riparian populations of the Brazilian Amazon. Int $J$ Occup Environ Health 2001; 7:23-25.

64. Lucotte M, Davidson R, Saint-Charles J, Guimarães JR. Human exposure to mercury as a consequence of landscape management and socio-economical behaviors. Part I: The Brazilian Amazon case study. RMZ Materials and Geoenvironment 2004; 51:668-672.

65. Roulet M, Lucotte M, Serique G, Coelho H, Sousa Passos CJ, Jesus da Silva E, Scavone de Andrade P, Mergler D, Guimarães JRD, Amorim M. Effects of recent human colonization on the presence of mercury in Amazonian ecosystems. Water, Air, and Soil Pollution 1999; 112(3-4):297-313.

66. Sampaio da Silva D, Lucotte M, Roulet M, Poirier H, Mergler D, Oliveira Santos E, Crossa M. Trophic structure and bioaccumulation of mercury in fish of 3 natural lakes of the Brazilian Amazon. Water, Air and Soil Pollution 2005; 165(1-4):77-94.

67. Mertens F, Saint-Charles J, Mergler D. Social communication network analysis of the role of participatory research in the adoption of new fish consumption behaviors. Soc Sci Med 2011; 75(4):643-650.

68. Fillion M, Passos CJ, Lemire M, Mertens F, Guimarães JRD, Philibert A, Mergler D. Neurotoxic sequelae of mercury exposure: an intervention and follow-up study in the Brazilian Amazon. EcoHealth 2012; 8(2):210-222.

69. Passos CJS, Mergler D, Fillion M, Lemire M, Mertens F, Guimarães JRD, Philibert A. Epidemiologic confirmation that fruit consumption influences mercury exposure in riparian communities in the Brazilian Amazon. Environ Res 2007; 105(2):183-193.

70. Joshi DD, Joshi AB, Joshi H. Epidemiology of echinococcosis in Nepal. South-East Asian J Trop Med Hyg 1997; 28(Supl.):26-31.

71. Waltner-Toews D, Kay JJ, Murray T, Neudoerffer C. Adaptive Methodology for Ecosystem Sustainability and Health (AMESH): an introduction. In: Midgley G, Ochoa-Arias AE, organizadores. Community operational research: systems thinking for community development. New York: Plenum, Kluwer Academic; 2004. p. 317-349.

72. Joshi DD, Sharma M, Waltner-Toews D. Rebuilding urban ecosystems for better community health in Kathmandu. In: Charron D, organizador. Ecohealth research in practice: Innovative applications of an ecosystem approach to health. Ottawa: International Development Research Centre; 2012. p. 191-201.

73. Joshi DD, Sharma M. An urban ecosystem health approach to make a cleaner city and better health in kathmandu, Nepal; 2006.

74. Dale S. Santé un approche écosystémique: Améliorer l'hygiène et la santé à Katmandou. Otawa: Centre de recherches pour le développement international; 2003.

75. Waltner-Toews D. An ecosystem approach to health and its applications to tropical and emerging diseases. Cad Saude Publica 2001; 17(Supl.):7-36.

Artigo apresentado em 17/03/2012

Aprovado em 27/04/2012

Versão final apresentada em 19/05/2012 